\title{
MUSGOS PLEUROCÁRPICOS DE MATA SERRANA EM PERNAMBUCO, BRASIL ${ }^{1}$
}

\author{
Jurandir A. Valdevino ${ }^{2}$ \\ Patrícia Sheyla A. Sá ${ }^{2}$ \\ Kátia Cavalcanti Pôrto ${ }^{3}$
}

Recebido em 19/03/2001. Aceito em 15/09/2001.

RESUMO - (Musgos Pleurocárpicos de Mata Serrana em Pernambuco, Brasil). Foi realizado o inventário dos musgos pleurocárpicos em uma área de Floresta Estacional Subcaducifólia Tropical Pluvionebular (mata serrana de brejo de altitude) situada na propriedade Bituri Grande, município de Brejo da Madre de Deus ( $8^{\circ} 08^{\prime} 45^{\prime}$ 'S, $\left.36^{\circ} 22^{\prime} 16^{\prime \prime} \mathrm{W}\right)$, Pernambuco. A propriedade possui ca. 700ha e situa-se a 900-1.120m s.m. A brioflora dos musgos pleurocárpicos é composta por 23 espécies pertencentes a 13 famílias. Constituem-se novos registros para a região Nordeste do Brasil: Aptychopsis subpungifolia (Broth.) Broth., Erytrodontium longisetum (Hook.) Par., Porothamnium flagelliferum (Hampe) Fleisch., Sematophyllum beyrichii (Hornsch.) Broth., S. galipense (C. Muell.) Mitt. e Trichosteleum glaziovii (Hampe) W.R. Buck. Para os táxons de ocorrência nova são apresentadas descrições, ilustrações, comentários e distribuição geográfica no Brasil.

Palavras-chave - musgos pleurocárpicos, floresta pluvionebular, brioflora

ABSTRACT - (Pleurocarpous mosses of a Sub-montane Rain Forest in Pernambuco State, Brazil). A floristic survey of bryophytes from a seasonal subdeciduous tropical forest was carried out in a remnant located in municipality of Brejo da Madre de Deus, State of Pernambuco ( $8^{\circ} 08^{\prime} 45^{\prime}$ 'S, 36 $22^{\prime} 16^{\prime \prime} \mathrm{W}$ ). The property has an area of 700ha, distributed over valleys and hills from $900-1120$ m altitude. Twenty three species belonging to 13 families of pleurocarpous mosses were registered. New references for Northeast region of Brazil are Aptychopsis subpungifolia (Broth.) Broth., Erytrodontium longisetum (Hook.) Par., Porothamnium flagelliferum (Hampe) Fleisch., Sematophyllum beyrichii (Hornsch.) Broth., S. galipense (C. Muell.) Mitt. and Trichosteleum glaziovii (Hampe) W. R. Buck. Descriptions, illustrations, comments and geographical distributions are given for each new record.

Key words - pleurocarpous mosses, sub-montane rain forest, bryoflora

1 Parte da dissertação de Mestrado do primeiro autor, auxílio CNPq.

2 Mestre em Criptógamos, UFPE (Universidade Federal de Pernambuco).

3 Dept $^{\mathrm{o}}$ de Botânica, Centro de Ciências Biológicas, UFPE, Av. Prof. Moraes Rego, s/n., Recife-PE, Brasil. 50670901.e-mail: kporto@npd.ufpe.br 


\section{Introdução}

No Nordeste brasileiro ocorrem áreas úmidas com cobertura de floresta estacional subcaducifólia tropical pluvionebular, inseridas no domínio das caatingas, denominadas "matas serranas de brejo de altitude". São condicionantes principais destas formações, a altitude superior a $600 \mathrm{~m}$ e a exposição (barlavento) a ventos de sudeste, que possibilitam comparativamente às áreas circundantes, redução de temperatura e umidade relativa, além de precipitação mais elevada. Por sua vez, os solos são mais desenvolvidos e profundos e a topografia favorece a formação de lagos e vales fluviais (Andrade-Lima 1960, 1961, 1970).

Briófitas ocorrentes em áreas de mata serrana de Pernambuco são referidas, principalmente, nos trabalhos de Yano \& Andrade-Lima (1987), Pôrto (1990, 1992) e Pôrto et al. (1999, 2000), que destacam a riqueza de representantes neste ambiente.

Visando dar continuidade ao conhecimento da composição briofítica de mata serrana, foi realizado o inventário de musgos em um remanescente florestal localizado no município de Brejo da Madre de Deus, Pernambuco. Registrou-se um total de 65 espécies, das quais 23 pertencem ao grupo artificial dos pleurocárpicos e encontram-se relacionadas neste artigo.

\section{Material e métodos}

A área de estudo está inserida na propriedade particular Bituri Grande, localizada no município de Brejo da Madre de Deus ( $8^{\circ} 08^{\prime} 45^{\prime}$ 'S, $36^{\circ} 22^{\prime} 16^{\prime}$ 'W), Pernambuco. A sede do município tem altitude de $627 \mathrm{~m}$ e valores pluviométricos médios anuais de $850,2 \mathrm{~mm}$, com estação chuvosa entre março a julho e estação seca de agosto a fevereiro (SUDENE 1990). A propriedade Bituri Grande ocupa cerca de 700ha e tem altitude de 900$1.120 \mathrm{~m}$. A cobertura vegetal é de floresta es- tacional subcaducifolia tropical pluvionebular, constituída por disjunções de matas remanescentes da cobertura primitiva (Andrade-Lima 1966). Estudos sobre a estrutura da vegetação e a diversidade fanerogâmica da área foram realizados por Lyra (1984a e b) e mais recentemente, complementados por $\mathrm{Sa}$ les et al. (1998), que incluem também as criptógamas vasculares.

As amostras de briófitas coletadas foram retiradas da base de tronco vivo, até $2 \mathrm{~m}$ de alt., tronco em decomposição, solo e rocha. A herborização e a preservação das amostras foram baseadas em Yano (1984). As exsicatas encontram-se depositadas no Herbário UFP, da Universidade Federal de Pernambuco, Recife.

As famílias encontram-se listadas em ordem alfabética. Nos estudos florísticos para a identificação das espécies foram utilizadas chaves analíticas contidas, principalmente, nos trabalhos de Florschütz (1964), Sehnem (1978), Griffin-III \& Morales (1983), Sharp et al. (1994) e Buck (1998). Para todos os táxons estudados são apresentadas indicações bibliográficas de descrição e ilustração, comentários de caracteres relevantes à identificação e distribuição geográfica no Brasil. Para as espécies de ocorrência nova no Nordeste (*) foram acrescidas descrições e ilustrações, elaboradas com base no material examinado, e quando necessário, completadas com dados de literatura.

\section{Resultados e discussão}

\section{BRACHYTHECIACEAE}

Rhynchostegium scariosum (Tayl.) A. Jaeg. Ber. Thätigk. St. Gallischen Naturwiss. Ges. 187677: 374. 1878.

Descrição e ilustração: Sharp et al. (1994). Material examinado: BRASIL. Pernambuco: Brejo da Madre de Deus, 15/IX/1992, J.A. Valdevino (UFP 9620); ibid., 21/X/1992, J.A. Val- 
devino (UFP 9621); (UFP 9610); ibid., 21/X/ 1992, K.C. Pôrto (UFP 9623).

Distribuição geográfica: RJ (Yano 1981); PE (Pôrto 1990); SP (Yano \& Santos 1993).

Comentários: Rhynchostegium scariosum é caracterizada por apresentar filídios ovalado-lanceolados, de margem serreada, costa simples até a metade da lâmina e células basais subquadráticas. Foi coletada sobre troncos vivos e mortos, em locais sombreados no interior da mata e em locais abertos, na margem.

\section{CRYPHAEACEAE}

Schoenobryum concavifolium (Griff.) Gang., Mosses E. India 5: 1209. 1976.

Descrição e ilustração: Buck (1998).

Material examinado: BRASIL. Pernambuco: Brejo da Madre de Deus, 3/XII/1992, J.A. Valdevino (UFP 9120); ibid., 27/IV/1992, J.A. Valdevino (UFP 9121); (UFP 9122).

Distribuição geográfica: MG, PR, RJ, RS, SC, SP (Yano 1981); PE (Yano \& Andrade-Lima 1987); RO (Yano 1989); ES (Schäfer-Verwimp 1991).

Comentários: Schoenobryum concavifolium caracteriza-se por apresentar filídios ovalados, de margem recurvada e costa única atingindo a metade da lâmina. Foi encontrada formando pequenos tapetes sobre troncos vivos, no interior da mata e em local aberto na margem.

\section{ENTODONTACEAE}

Entodon beyrichii (Schwaegr.) C. Muell., Linnaea 18: 708. 1845.

Descrição e ilustração: Buck (1998).

Material examinado: BRASIL. Pernambuco: Brejo da Madre de Deus, 14/IX/1992, J.A. Valdevino (UFP 9615); ibid., 21/IX/1992, J.A. Valdevino (UFP 9614, 9617).

Distribuição geográfica: PR (Kummrow \& Prevedello 1982); MT (Yano 1989); ES (SchäferVerwimp 1991); SP (Yano \& Santos 1993); RJ (Oliveira e Silva 1998); RS (Lemos-Michel 1999) e PE (Pôrto et al. 2000).
Comentários: Entodon beyrichii caracteriza-se por apresentar filídios lanceolados, com costa dupla restrita à base e esporófito com seta vermelha. Foi encontrada sobre tronco morto e rocha, em locais sombrios e úmidos no interior da mata. Cresce formando pequenos tapetes, associada a Erythrodontium longisetum e Racopilum tomentosum.

*Erythrodontium longisetum (Hook.) Par., Index Bryol. 436. 1896.

Fig. 1-6

Gametófito verde-claro, brilhante, ca. $10 \mathrm{~mm}$ compr., irregularmente pinado ou subpinado, ramos ascendentes juláceos. Filídios oblongo-ovalados, ca.1,0-1,5 x 0,8-1,0mm, fracamente côncavos, às vezes plicados, imbricados quando secos, margem inteira, ápice curto apiculado, levemente serreado. Costa ausente, ou raramente dupla e restrita à base. Células alares numerosas, subquadráticas a retangulares, ca. $10 \times 15 \mu \mathrm{m}$, lisas, as medianas lineares, ca. 75$95 \times 7 \mu \mathrm{m}$, lisas. Seta ca. $10 \mathrm{~mm}$ compr., amarela. Cápsula oblongo-cilíndrica, ca. 2-5mm compr., castanho-claro, ereta. Peristômio duplo, exostômio liso ou estriado, endostômio rudimentar, liso.

Material examinado: BRASIL. Pernambuco: Brejo da Madre de Deus, 15/III/1992, J.A. Valdevino (UFP 9624); ibid., 22/X/1992, J.A. Valdevino (UFP 9616); ibid., 21/X/1992, K.C. Pôrto (UFP 9613); ibid., 21/X/1992, J.A. Valdevino (UFP 9617).

Distribuição geográfica: MG, RS, SP (Yano 1981); RJ (Yano 1989) e PE.

Comentários: Erythrodontium longisetum caracteriza-se por apresentar filídios oblongo-ovalados e seta amarela. Esta espécie é próxima a Entodon beyrichii, que apresenta filídios lanceolados, bicostados, e seta vermelha. Cresce formando pequenos tapetes sobre troncos vivos e mortos e rochas, em ambiente sombrio e úmido no interior da mata. Foi encontrada em associação com Brachymenium morasicum Besch., 
Entodon beyrichii e Groutiella tomentosa (Hornsch.) Wijk. \& Marg.

\section{FABRONIACEAE}

Fabronia ciliaris (Brid.) Brid. var. polycarpa (Hook.) Buck, Brittonia 35: 251.1983.

Descrição e ilustração: Sharp et al. (1994). Material examinado: BRASIL. Pernambuco: Brejo da Madre de Deus, 22/X/1992, J.A. Valdevino (UFP 9110); (UFP 9111); (UFP 8498); 21/X/1992, K.C. Pôrto (UFP 8501); ibid., 3/XII/ 1992, J. A. Valdevino (UFP 9112).

Distribuição geográfica: BA, CE, MG, PE, PR, RJ, RS, SC, SP (Yano 1981); ES (SchäferVerwimp 1991).

Comentários: Fabronia ciliaris var. polycarpa caracteriza-se por apresentar filídios lanceolados, longo-acuminados, fortemente denticulados, costa simples até a metade da lâmina e células medianas lineares e as basais subquadráticas. Foi encontrada crescendo sobre troncos vivos, principalmente nos fustes de jaqueira ( $A r$ throcarpus integrifolia L.) em local aberto, na margem da mata e sobre troncos mortos e solo, em ambiente sombrio e úmido no interior da mata. Cresce formando pequenos tapetes, associada a Barbula indica (Hook.) Spreng. ex Stend., Brachymenium morasicum Besch., Entodontopsis leucostega e Philonotis uncinata (Schwaegr.) Brid.

\section{HOOKERIACEAE}

Callicostella pallida (Hornsch.) Angstr., Öfvers. Förh. Kongl. Svenska Vetensk.-Akad. 33(4): 27. 1876.

Descrição e ilustração: Buck (1998).

Material examinado: BRASIL. Pernambuco: Brejo da Madre de Deus, 28/V/1992, J.A. Valdevino (UFP 8484); ibid., 15/IX/1992, J.A. Valdevino (UFP 8483); (UFP 9107); ibid., 29/V/ 1992, J.A. Valdevino (UFP 8484); ibid., 22/X/ 1992, J. A. Valdevino (UFP 9108); (UFP 9109). Distribuição geográfica: AM, GO, MG, MT, PA, RJ, SP (Yano 1981); AP (Yano \& Lisboa 1988);
PR, RS (Yano 1989); PE (Pôrto 1990); ES (Schäfer-Verwimp 1991); RR (Yano \& Mello 1992).

Comentários: Callicostella pallida é reconhecida facilmente pelos filídios com costa dupla, atingindo até próximo ao ápice, margem irregularmente serreada, e pelas células medianas unipapilosas. Foi coletada sobre troncos vivos, mortos e rocha, em ambiente sombrio e úmido no interior da mata, e próximo e dentro de riacho.

\section{HYPNACEAE}

Isopterygium tenerum (Sw.) Mitt., J. Linn. Soc. Lond. Bot. 12: 499. 1869.

Descrição e ilustração: Ireland (1992).

Material examinado: BRASIL. Pernambuco: Brejo da Madre de Deus, 15/IX/1992, tronco vivo, J.A. Valdevino (UFP 9114); (UFP 9115). Distribuição geográfica: AM, GO, PA, PR, RS, SC, SP (Yano 1981); PB (Marinho 1987); MT (Yano 1989); PE (Pôrto 1990); ES (SchäferVerwimp 1991); RR (Yano \& Mello 1992); AC, BA, MG (Yano 1995).

Comentários: Isopterygium tenerum caracteriza-se por apresentar filídios ovalado-lanceolados, ápice longo-acuminado, costa ausente e células medianas lineares, lisas. Foi encontrada formando tapetes sobre troncos vivos, em ambiente sombrio no interior da mata.

\section{METEORIACEAE}

Pilotrichella versicolor (C. Muell.) Jaeg., Ber. St. Gall. Natur. Ges. 1875-76: 258. 1877. Descrição e ilustração: Florschütz (1964). Material examinado: BRASIL. Pernambuco: Brejo da Madre de Deus, 28/III/1992, J.A. Valdevino (UFP 9089); ibid., 21/X/1992, J.A. Valdevino (UFP 9100); ibid., 27/IV/1992, J.A. Valdevino (UFP 9102).

Distribuição geográfica: RJ, RS, SC, SP (Yano 1981); PE (Yano \& Andrade-Lima 1987); RO (Lisboa \& Yano 1987); ES (Schäfer-Verwimp 1991). 
Comentários: Pilotrichella versicolor caracteriza-se por apresentar ramos secundários longo-pendentes, filídios ovalados, côncavos na base, ápice acuminado e costa simples até a metade da lâmina. Foi encontrada sobre troncos vivos, em ambiente sombrio e úmido no interior da mata, em associação com Jaegerina scariosa e Squamidium leucotrichum.

Squamidium leucotrichum (Tayl.) Broth., Nat. Pflanzenfam. 1(3): 809. 1906.

Descrição e ilustração: Buck (1998).

Material examinado: BRASIL. Pernambuco: Brejo da Madre de Deus, 15/III/1992, J.A. Valdevino (UFP 9105); ibid., 3/XII/1992, J.A. Valdevino (UFP 9104); ibid., 27/IV/1993, J.A. Valdevino (UFP 9103).

Distribuição geográfica: AL, MG, PR, RJ, SC, SP (Yano 1981); PE (Yano \& Andrade-Lima 1987); AM (Yano 1989); RS ( Lemos-Michel 1999); BA (Yano \& Bastos 1994/1995).

Comentários: Squamidium leucotrichum caracteriza-se por apresentar ramos secundários pendentes, com filídios oblongo-lanceolados, serreados, levemente côncavos e ápice longo-acuminado. Foi encontrada crescendo sobre troncos vivos e mortos, em ambiente sombrio e úmido, no interior da mata.

\section{NECKERACEAE}

*Porothamnium flagelliferum (Hampe) Fleisch., Nat. Pflanzenfam. Sér. 2,11(2): 199. 1925. Fig. 7-11

Gametófito frondoso, dendróide, ramos secundários pinados até bipinados, geralmente pendentes. Filídios complanados, ovalado-oblongos, ca. 1-1,5mm compr., ápice mucronado, fortemente denticulado. Costa simples, alcançando $2 / 3$ da lâmina. Células da base hexagonais, ca. $15-30 \times 10 \mu \mathrm{m}$, as medianas fusiformes, ca. $60-80 \mu \mathrm{m}$ compr., lisas, paredes espessadas; as apicais ovalado-romboidais, ca. 10-20 $\mu \mathrm{m}$ compr. Esporófito não estudado.
Material examinado: BRASIL. Pernambuco: Brejo da Madre de Deus, 21/X/1992, K.C. Pôrto (UFP 9116).

Distribuição geográfica: MG, SC (Yano 1981); RS (Yano 1989) e PE.

Comentários: Porothamnium flagelliferum é caracterizada por apresentar ramos curto-pendentes, filídios complanados, com ápice denticulado e células medianas fusiformes, lisas, de paredes espessadas. Foi encontrada sobre tronco vivo, em ambiente sombrio no interior da mata.

\section{PHYLLOGONIACEAE}

Phyllogonium viride Brid., Bryol. Univ. 2: 273. 1827.

Descrição e ilustração: Yano \& Mello (1989). Material examinado: BRASIL. Pernambuco: Brejo da Madre de Deus, 28/III/1992, J.A. Valdevino (UFP 9117); ibid., 28/IV/1992, J.A. Valdevino (UFP 9118).

Distribuição geográfica: MG, RJ, RS, SC, SP (Yano 1981); PE (Yano \& Andrade-Lima 1987); ES (Schäfer-Verwimp 1991); BA, PR (Yano \& Mello 1992); AL (Yano 1995).

Comentários: Phyllogonium viride caracterizase por apresentar hábito pendente, filídios côncavo-naviculares, ápice recurvado e células medianas lineares. Destaca-se pela exuberância do hábito longo-pendente, que se ramifica a partir dos ramos superiores das árvores. Foi encontrada crescendo sobre troncos vivos, em ambiente sombrio no interior da mata.

\section{PTEROBRYACEAE}

Henicodium geniculatum (Mitt.) Buck, Bryol. 92 (4): 534. 1989.

Descrição e ilustração: Buck (1998).

Material examinado: BRASIL. Pernambuco: Brejo da Madre de Deus, 13/IX/1992, J.A. Valdevino (UFP 9082); ibid., 28/V/1992, J.A. Valdevino (UFP 9083); (UFP 9089); ibid., 29/V/ 1992, J.A. Valdevino (UFP 9084); 14/IX/1991, J.A. Valdevino (UFP 9085); ibid., 21/X/1992, K.C. Pôrto (UFP 9086); (UFP 9088). 

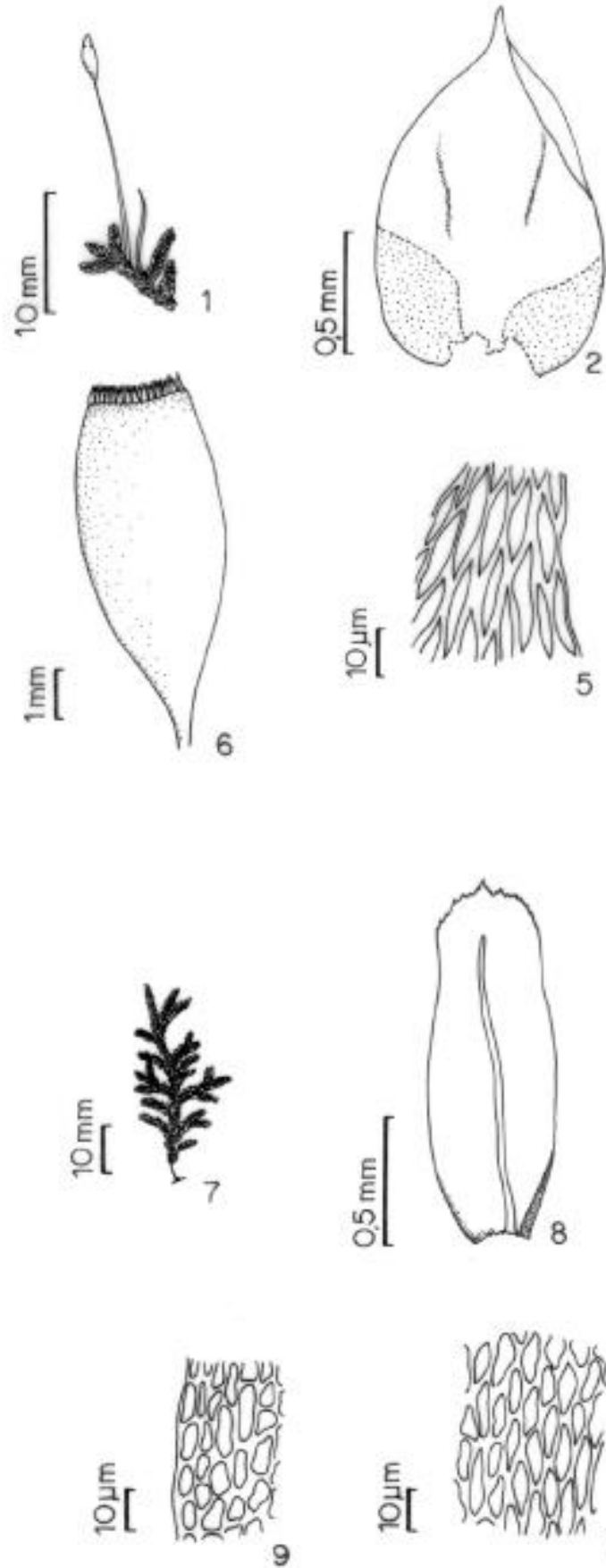
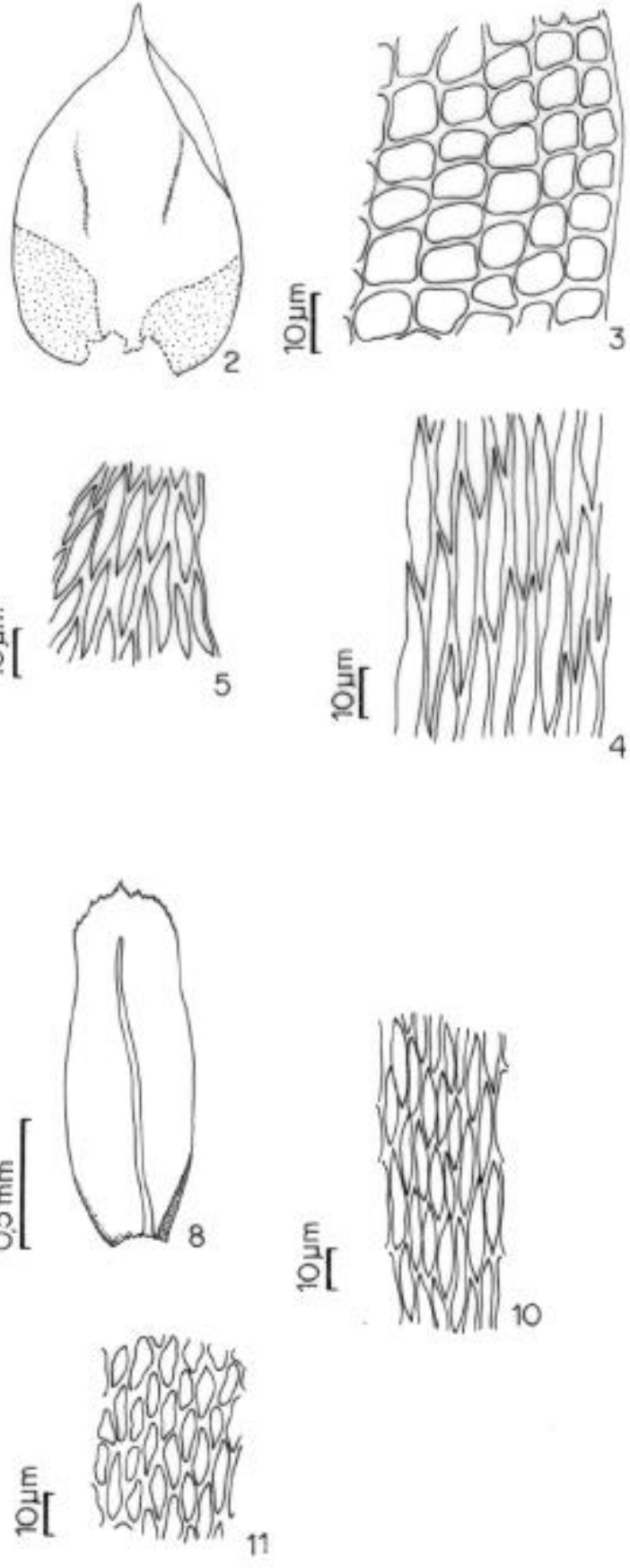

Figuras 1-6. Erythodontium longisetum (Hook.) Par. 1. hábito; 2. filídio; 3. células marginais da base; 4. células medianas; 5. células apicais; 6. cápsula. 7-11. Porothamnium flagelliferum (Hampe) Fleisch. 7. hábito; 8. filídio; 9. células da base; 10 . células medianas; 11 . células apicais. 
Distribuição geográfica: AL, AM, GO, MT, PA, RJ, SP (Yano 1981); AP (Yano \& Lisboa 1988); ES (Schäfer-Verwimp 1991); BA (Yano \& Bastos 1994/1995); PE (Yano 1995).

Comentários: Henicodium geniculatum é caracterizada pelos filídios ovalado-lanceolados, de margem recurvada e serreada, costa simples, percurrente e células medianas papilosas. Foi encontrada crescendo sobre troncos vivos, em ambiente sombrio no interior da mata.

Jaegerina scariosa (Lor.) Arz., Amer. Midl. Nat. 52: 12.1954.

Descrição e ilustração: Buck (1998).

Material examinado: BRASIL. Pernambuco: Brejo da Madre de Deus, 21/X/1992, K.C. Pôrto (UFP 9119); ibid., 3/XII/1992, J.A. Valdevino (UFP 9120).

Distribuição geográfica: GO, MT, PA, RJ, SP (Yano 1981); PE (Yano \& Andrade-Lima 1987); AL (Yano 1989); ES (Schäfer-Verwimp 1991); MG (Yano 1992).

Comentários: Jaegerina scariosa caracteriza-se por apresentar filídios ovalados, levemente côncavos, costa única atingindo até a metade da lâmina e células medianas lineares, lisas. Foi encontrada sobre troncos vivos, em ambiente sombrio no interior da mata, crescendo em pequenas colônias, associada a Pilotrichella versicolor.

\section{RACOPILACEAE}

Racopilum tomentosum (Sw. ex Hedw.) Brid., Bryol. Univ. 2: 719. 1827.

Descrição e ilustração: Sharp et al. (1994).

Material examinado: BRASIL. Pernambuco: Brejo da Madre de Deus, 28/V/1992, J.A. Valdevino (UFP 9090); ibid., 29/V/1992, J.A. Valdevino (UFP 9091); ibid., 14/IX/1992, J.A. Valdevino (UFP 9092); ibid., 15/IX/1992, J.A. Valdevino (UFP 9003); ibid., 21/X/1992, J.A. Valdevino (UFP 9094); (UFP 9095); ibid., 21/X/ 1992, K.C. Pôrto (UFP 9096); (UFP 9097).

Distribuição geográfica: AM, GO, MT, MG, PA, RJ, RS, SC, SP (Yano 1981); PE (Yano \& An-
drade-Lima 1987); BA, DF, RO (Yano 1989); ES (Schäfer-Verwimp 1991); CE (Yano \& Costa 1992).

Comentários: Racopilum tomentosum é facilmente reconhecida pelos filídios oblongos, dispostos em fileiras opostas, ápice serreado, costa simples, excurrente e células medianas sub-quadráticas. Cresce sobre troncos vivos e mortos, solo e rochas, em ambiente sombrio e úmido, na margem e no interior da mata. Foi encontrada formando grandes colônias, associada a Bryum densifolium Brid., Entodon beyrichii, Rhynchostegium scariosum e Squamidium leucotrichum.

\section{SEMATOPHYLLACEAE}

*Aptychopsis subpungifolia (Broth.) Broth., Nat. Pflanzenfam. Sér. 2, 11(2): 411. 1925.

Fig. 12-16

Gametófito verde-claro a amarelo-dourado, brilhante, ramos secundários ascendentes, ca. 10-20mm compr. Filídios lanceolados, ca. 1,5-2,0 x 0,3mm, margem inteira, ápice acuminado. Costa ausente. Células alares 4-5, oblongas a retangulares, ca. 30 × $20 \mu \mathrm{m}$, paredes espessadas; células supra-alares numerosas, subquadráticas; as medianas alongadas, ca. 70 x $100 \mu \mathrm{m}$, lisas, paredes delgadas. Filídios periqueciais subulados. Seta lisa, ca. 5$7 \mathrm{~mm}$ compr., vermelha. Cápsula ovalado-cilíndrica, fracamente inclinada, ca. $1 \mathrm{~mm}$ compr., castanho-claro, peristômio duplo, exostômio estriado, endostômio de segmentos curtos, sem cílios.

Material examinado: BRASIL. Pernambuco: Brejo da Madre de Deus, 29/V/1992, J. A. Valdevino (UFP 8740); ibid., 3/XII/1992, J.A. Valdevino (UFP 8737); (UFP 8736).

Distribuição geográfica: MG, SC (Sehnem 1978) e PE.

Comentários: Aptychopsis subpungifolia apresenta células alares de paredes espessas e filídios periqueciais subulados, características estas que a diferenciam facilmente das demais espé- 
cies do gênero. Foi encontrada sobre troncos vivos, em ambiente sombrio e úmido, no interior da mata e a margem de riacho.

Meiothecium revolubile Mitt., J. Linn. Soc. Bot. 12: 471.1869.

Descrição e ilustração: Churchill \& Linares (1995).

Material examinado: BRASIL. Pernambuco:

Brejo da Madre de Deus, 13/X/1991, J.A. Valdevino (UFP 9176).

Distribuição geográfica: AM, MG (Yano 1981); ES (Behar et al. 1992); PE (Pôrto et al. 1993). Comentários: Meiothecium revolubile é reconhecida por apresentar filídios ovalado-lanceolados, com ápice levemente serreado e células alares ovaladas, infladas, paredes espessas, amarelas; as medianas ovalado-oblongas a lineares, cápsula ovalada-cilíndrica, com peristômio simples. Foi encontrada formando extensos tapetes, crescendo sobre tronco vivo, em ambiente sombrio e úmido no interior da mata.

*Sematophyllum beyrichii (Hornsch.) Broth., Nat. Pflanzenfam. Sér. 2, 11(2): 432. 1925.

Fig. 17-21

Gametófito verde-claro a verde-amarelado, brilhante, ramos irregularmente pinados, ascendentes, ca. 10mm compr. Filídios ereto-patentes, lanceolados, ca. 1,5-2,0 x 0,5mm, fracamente côncavos, ápice longo-acuminado, margem inteira. Costa ausente. Células alares 2-3, oblon-

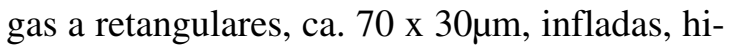
alinas; as supra-alares sub-quadráticas, numerosas, amarelas; células da base ovalado-lineares, ca. $35-55 \mu \mathrm{m}$ compr., as medianas lineares ca. 70-95 $\mu \mathrm{m}$ compr., lisas, ovaladas próximo ao ápice. Filídios periqueciais longo-acuminados. Seta ca. 15-20mm compr., vermelha, torcida quando seca. Cápsula oblonga, ca. 0,7-1,0mm compr., inclinada, castanho-escuro. Peristômio duplo.

Material examinado: BRASIL. Pernambuco: Brejo da Madre de Deus, 13/XI/1991, J.A. Val- devino (UFP 9178); (UFP 9170); ibid., 28/III/ 1992, J.A. Valdevino (UFP 9162); ibid., 28/V/ 1992, J.A. Valdevino (UFP 8739); ibid., 21/X/ 1992, K.C. Pôrto (UFP 9165).

Distribuição geográfica: PR, RJ, RS, SP (Yano 1981); ES (Schäfer-Verwimp 1991) e PE.

Comentários: Sematophyllum beyrichii caracteriza-se pelos filídios ereto-patentes, lanceolados, longo-acuminados, os periqueciais com ápice quase filiforme. Foi encontrada formando pequenos tapetes sobre troncos vivos e mortos, em ambiente sombrio no interior da mata.

*Sematophyllum galipense (C. Muell.) Mitt., J. Linn. Soc. Lond. Bot. 12: 480. 1869.

Fig. 22-26

Gametófito verde-claro a verde-amarelado, brilhante, ramos secundários ascendentes, irregulares, pinados. Filídios lanceolados, ca. 2,0 x 0,6mm compr., côncavos, ápice acuminado, margem inteira. Costa ausente. Células alares

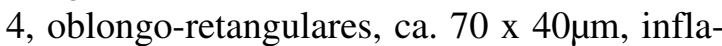
das, amarelas, as supra-alares subquadráticas, amarelas, numerosas, células basais lineares, ca. $55 \mu \mathrm{m}$ compr., geralmente amarelas, as medianas menores, ca. $35 \mu \mathrm{m}$ compr., lisas. Seta ca. 10mm compr., castanho-claro. Cápsula ovóide, inclinada, castanho-claro, ca. 0,5-1,0mm. Peristômio duplo, exostômio estriado, endostômio com segmentos perfurados e ciliados.

Material examinado: BRASIL. Pernambuco: Brejo da Madre de Deus, 29/V/1992, J.A. Valdevino (UFP 8741); ibid., 20/X/1992, J. A. Valdevino (UFP 9173).

Distribuição geográfica: GO, MT, MG, PA, RJ, RS, SP (Yano 1981); ES (Schäfer-Verwimp 1991); RO (Yano \& Mello 1992); RR (Yano 1995); BA (Bastos \& Vilas Bôas-Bastos 1998) e PE.

Comentários: Sematophyllum galipense caracteriza-se pelos filídios lanceolados, curto-acuminados, geralmente plicados e profundamente côncavos, as células alares são oblongo-retangulares, as supra-alares grandes e as apicais alon- 

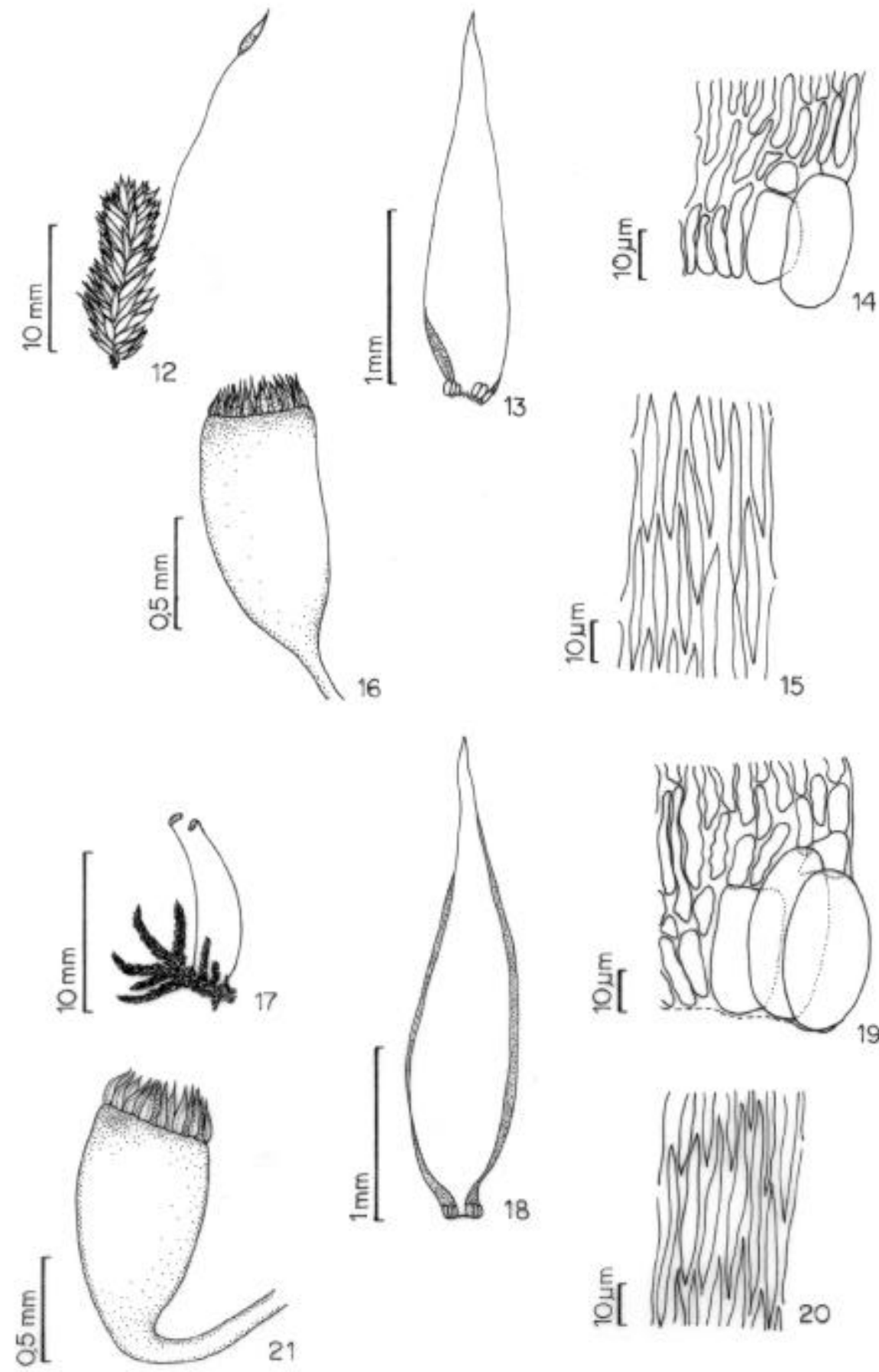

Figuras 12-16. Aptychopsis subpungifolia (Broth.) Broth. 12. hábito; 13. filídio; 14. células alares; 15. células medianas; 16. cápsula. 17-21. Sematophyllum beyrichii (Hornsch.) Broth. 17. hábito; 18. filídio; 19. células alares; 20. células medianas; 21 . cápsula com dentes. 
gadas. Foi encontrada formando pequenos tapetes sobre rochas, em ambiente sombrio e úmido, no interior da mata.

Sematophyllum subpinnatum (Brid.) Britt., Bryologist 21: 28. 1918.

Descrição e ilustração: Sharp et al. (1994). Material examinado: BRASIL. Pernambuco: Brejo da Madre de Deus, 22/X/1992, J.A. Valdevino (UFP 9173); ibid., 28/V/1992, J.A. Valdevino (UFP 9175).

Distribuição geográfica: AM, GO, MT, MG, PA, PR, PE, RJ, SC, SP (Yano 1981); PB (Marinho 1987); ES (Behar et al. 1992); AP, CE, RR (Yano 1995); BA (Vilas Bôas-Bastos \& Bastos 1998).

Comentários: Sematophyllum subpinnatum é caracterizada por apresentar os filídios oblongo-ovalados, curto-acuminados, células medianas oblongo-romboidais e as alares oblongas, verde-amareladas a avermelhadas. Cresce formando tapetes sobre tronco vivo e rocha, em ambiente sombrio e úmido no interior da mata.

Sematophyllum subsimplex (Hedw.) Mitt., J. Linn. Soc. Lond. Bot. 12: 494. 1869.

Descrição e ilustração: Sharp et al. (1994).

Material examinado: BRASIL. Pernambuco:

Brejo da Madre de Deus, 13/XI/1991, J.A. Valdevino (UFP 9172); ibid., 28/V/1992, J.A. Valdevino (UFP 9176); (UFP 9171); (UFP 8738); ibid., 25/IX/1992, J.A. Valdevino (UFP 9166); ibid., 21/X/1992, J.A. Valdevino (UFP 9169); (UFP 9169).

Distribuição geográfica: AM, DF, GO, RJ, SP (Yano 1981); PB (Marinho 1987); MA, MT, MG, PA, PR, SC (Yano 1989); PE (Pôrto 1990); ES (Schäfer-Verwimp 1991); AP, RS (Yano 1992); RR, SE (Yano 1995); PI (Castro 1997); BA (Vilas Bôas-Bastos \& Bastos 10998).

Comentários: Sematophyllum subsimplex caracteriza-se por apresentar filídios ovalados, côncavos, longo-acuminados, células medianas lineares e as alares infladas, amarelas. Foi encon- trada crescendo sobre troncos vivos e mortos, em ambiente sombrio e úmido no interior da mata, formando grandes tapetes, ocasionalmente associada a Bryum densifolium Brid. e Schoenobryum concavifolium.

*Trichosteleum glaziovii (Hampe) Buck, Nova Hedwigia 66: 243. 1998.

Fig. 27-32

Gametófito verde-claro a verde-amarelado, ramos secundários pinados. Filídios ovalado-lanceolados, ca. 1,0-1,5 x 0,2mm, secundos, falcados, ápice longo-acuminado e denteado. Costa ausente. Células alares 3-4, oblongo-retangulares, ca. 50 × $20 \mu \mathrm{m}$, paredes espessadas, infladas, amarelas, as supraalares subquadrátricas, células da base lineares, ca. 40-60 $\mu \mathrm{m}$ compr., amarelas, com paredes tuberculares, as medianas fusiformes ca. 70-95 $\mu \mathrm{m}$ compr., papilosas, paredes delgadas, as apicais menores ca. $20-30 \mu \mathrm{m}$ compr., papilosas exceto na margem. Seta lisa, ca. 10$15 \mathrm{~mm}$ compr., castanho-claro. Cápsula ovalado-cilíndrica, ca. $0,5 \mathrm{~mm}$ compr., inclinada, castanho-claro. Peristômio duplo, exostômio estriado abaixo e papiloso acima, endostômio liso ou papiloso.

Material examinado: BRASIL. Pernambuco: Brejo da Madre de Deus, 29/V/1992, J.A. Valdevino (UFP 8734); ibid., 21/X/1992, J.A. Valdevino (UFP 9164).

Distribuição geográfica: PR (Sehnem 1978); RJ (Yano 1981) e PE.

Comentários: Trichosteleum glaziovii apresenta como características marcantes os filídios secundos falcados, de ápice longamente acuminado. Foi encontrada formando pequenos tapetes sobre troncos vivos e mortos, em ambiente sombrio e úmido no interior da mata.

Trichosteleum papillosum (Hornsch.) Jaeg., Ber. S. Gall. Naturv. Ges. 176-77: 419. 1878. Descrição e Ilustração: Yano \& Lisboa (1988). 

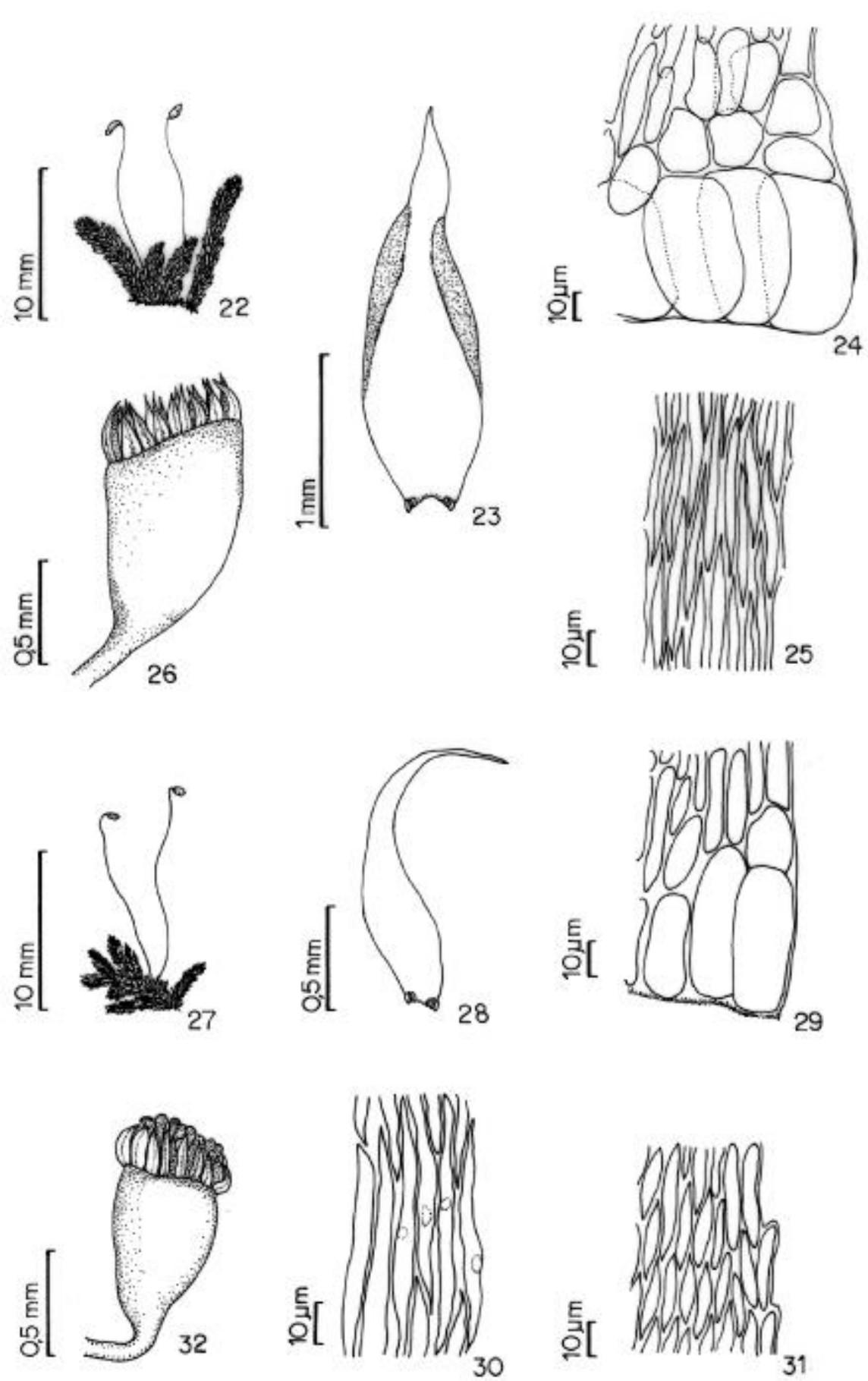

Figuras 22-26. Sematophyllum galipense (C. Muell.) Mitt. 22. hábito; 23. filídio; 24. células medianas; 25. células alares; 26. cápsula. 27-32. Trichosteleum glaziovii (Hampe) W.R. Buck. 27. hábito; 28. filídio; 29. células alares; 30. células medianas; 31 . células apicais marginais; 32. cápsula com dentes. 
Material examinado: BRASIL. Pernambuco: Brejo da Madre de Deus, 22/X/1992, J.A. Valdevino (UFP 8742).

Distribuição geográfica: AM, ES, MG, PA, RJ, SC, SP (Yano 1981); AP (Yano \& Lisboa 1988); RO (Yano \& Mello 1992); SE (Yano 1992); PE (Germano \& Pôrto 1997).

Comentários: Trichosteleum papillosum caracteriza-se por apresentar filídios longo-acuminados, ca. 1-1,2mm compr. e células apicais fusiformes, as medianas lineares, unipapilosas, com papilas grandes e centrais sobre o lúmen. Foi encontrada crescendo sobre rocha, em ambiente sombrio e úmido, no interior da mata próximo a um riacho.

\section{STEREOPHYLLACEAE}

Entodontopsis leucostega (Brid.) Buck \& Ireland, Nova Hedwigia 41: 103. 1985.

Descrição e ilustração: Buck \& Ireland (1985); Sharp et al. (1994).

Material examinado: BRASIL. Pernambuco: Brejo da Madre de Deus, 22/X/1992, J.A. Valdevino (UFP 9111); ibid., 14/IX/1992, J.A. Valdevino (UFP 9233)

Distribuição geográfica: AM, BA, CE, GO, MG, RJ (Yano 1981); PE (Vital et al. 1991); SP (Yano \& Santos 1993); PI (Ireland \& Buck 1994).

Comentários: Entodontopsis leucostega caracteriza-se por apresentar os filídios ovalado-lanceolados, acuminados, de margem serreada próximo ao ápice, costa simples até a metade da lâmina e células medianas lineares, lisas, as alares quadráticas a retangulares. Foi encontrada sobre rocha e solo, em ambiente sombrio e úmido no interior da mata, formando pequenos tapetes, ocasionalmente associada a Barbula indica (Hook.) Spreng. ex Stend., Brachymenium morasicum Besch., Fabronia ciliaris var. polycarpa e Philonotis uncinata (Schwaegr.) Brid.

\section{Agradecimentos}

Aos Drs. William R. Buck, do New York Botanical Garden (USA), Dana Griffin III, da
University of Florida (USA), Olga Yano, do Instituto de Botânica (SP), pelo auxílio na identificação de algumas amostras, e a Shirley R. Germano, Sylvia M. de Oliveira e Gláucia Nascimento, pela leitura e sugestões ao texto.

\section{Referências bibliográficas}

Andrade-Lima, D. 1960. Estudos fitogeográficos de Pernambuco. Arquivos do Instituto de Pesquisas Agrônomicas 5: 305-341.

Andrade-Lima, D. 1961. Tipos de florestas de Pernambuco. In: Anais da Associação dos Geográfos Brasileiro 12: 68-85.

Andrade-Lima, D. 1966. Esboço fitoecológico de alguns brejos de Pernambuco. Boletim Técnico do Instituto de Pesquisas Agronômicas 8: 3-7.

Andrade-Lima, D. 1970. Recursos vegetais de Pernambuco. Boletim Técnico do Instituto de Pesquisas Agronômicas 41: 1-32.

Bastos, C.J.P. \& Vilas Bôas-Bastos, S.B. 1998. Adições à brioflora (Bryopsida) do Estado da Bahia, Brasil. Tropical Bryology 15: 111-116.

Behar, L.; Yano, O. \& Vallandro, G.C. 1992. Briófitas da Restinga de Setiba, Guarapari, Espírito Santo. Boletim do Museu de Biologia Mello Leitão 1: 25-38.

Buck, W.R. 1998. Pleurocarpous Mosses of the West Indies. The New York Botanical Garden 82: 1-400.

Buck, W.R. \& Ireland, R.R. 1985. A reclassification of the Plagiotheciaceae. Nova Hedwigia 41: 89-125.

Castro, N.M.C.F. 1997. Bryopsida do Parque Nacional de Sete Cidades, Piauí, Brasil. Dissertação de Mestrado. Universidade Federal de Pernambuco, Recife.

Churchill, S.P. \& Linares C.E.L. 1995. Prodromus Bryologiae Novo Granatensis. Introduccion a la flora de musgos de Colombia. Bogota: Universidad Nacional de Colombia. 924 p.

Florschütz, P.A. 1964. The mosses of Suriname. E. J. Brill, Leiden 6: 1-127.

Germano, S.R. \& Pôrto, K.C. 1997. Floristic Survey of Epixylic Bryophytes of an Area Remnant of The Atlantic Forest (Timbaúba - PE, Brazil). 1 - Hepaticopsida (except Lejeuneaceae) and Bryopsida. Tropical Bryology 12: 21-28.

Griffin-III, D. \& Morales, M.I. 1983. Keys to the genera of mosses of Costa Rica. Brenesia 21: 299-323.

Ireland, R.R. 1992. The moss genus Isopterygium (Hypnaceae) in Latin American. Tropical Bryology 6: 111-132. 
Ireland, R.R. \& Buck, W.R. 1994. Stereophyllaceae. Flora Neotropica, monografh 65: 1-49.

Kummrow, R. \& Prevedello, S.M. 1982. Lista de musgos paranaenses do Museu Botânico municipal. Boletim do Museu Botânico Municipal Curitiba 54: 1-36.

Lemos-Michel, E. 1999. Briófitas epífitas sobre Araucaria angustifolia (Bert.) Kuntze no Rio Grande do Sul, Brasil. Tese de Doutorado. Universidade de São Paulo, São Paulo.

Lisboa, R.C.L. \& Yano, O. 1987. Novas ocorrências de briófitas na Amazônia brasileira. Boletim do Museu Paraense Emílio Goeldi, Botânica 3(2): 141156.

Lyra, A.L.R.T. 1984a. Efeito do relevo na vegetação de duas áreas do município do Brejo da Madre de Deus (PE). II. Estrutura da vegetação. Pp. 279-285. In: Anais do XXXIV Congresso Nacional de Botânica, Porto Alegre, 1992. Sociedade Botânica do Brasil.

Lyra, A.L.R.T. 1984b. Efeito do relevo na vegetação de duas áreas do município do Brejo da Madre de Deus (PE). III. Diversidade florística. Pp. 287-296. In: Anais do XXXIV Congresso Nacional de Botânica, Porto Alegre, 1992. Sociedade Botânica do Brasil.

Marinho, M.G.V. 1987. Bryopsida na Reserva Florestal do IBDF, João Pessoa, Paraíba, Brasil. Dissertação de Mestrado. Universidade Federal de Pernambuco, Recife.

Oliveira e Silva, M.I.M.N. 1998. Brófitas da Reserva Ecológica de Rio das Pedras, município de Mangaratiba, do Parque Estadual da Ilha Grande e da Reserva Biológica Estadual da Praia do Sul, município de Angra dos Reis, estado do Rio de Janeiro. Tese de Doutorado. Universidade de São Paulo, São Paulo.

Pôrto, K.C. 1990. Bryoflores d'une forêt de plaine et d'une forêt d'altitude moyenne dans 1'Etat de Pernambuco (Brésil): Analyse floristique. Cryptogamie, Bryologie Lichénologie 11(2): 109-161.

Pôrto, K.C.1992. Bryoflores d'une forêt de plaine et d'une forêt d'altitude moyenne dans 1'Etat de Pernambuco (Brésil): Analyse écologique comparative des forêts. Cryptogamie, Bryologie Lichénologie 13(3): 187-219.

Pôrto, K.C.; Belo, M.M.A.; Fonseca, E.R. \& Silva, E.C. 1993. Brioflora da Reserva de Gurjaú (Cabo-PE). Biologica Brasilica 5(1/2): 27-42.

Pôrto, K.C.; Germano, S.R. \& Oliveira, S.M. 2000. New records of bryophytes for Pernambuco State, Brazil. Tropical Bryology 18: 107-114.

Pôrto, K. C.; Gradstein, S.R.; Yano, O.; Germano, S.R.
\& Costa, D.P. 1999. New and interesting records of Brazilian bryophytes. Tropical Bryology 17: 39-45.

Sales, M.F.; Mayo, S.J. \& Rodal, M.J.N. 1998. Plantas vasculares das florestas serranas de Pernambuco: um checklist da flora ameaçada dos brejos de altitude, Pernambuco, Brasil. Recife: Universidade Federal Rural de Pernambuco. 130p.

Schäfer-Verwimp, A. 1991. Contribution to the knowledge of the bryophyte flora of Espírito Santo, Brazil. The Journal of the Hattori Botanical Laboratory 69: 147-170.

Sehnem, A. 1978. Musgos Sul-brasileiros. VI. Pesquisas, série Botânica 32: 1-70.

Sharp, A.J.; Crum, H. \& Eckel, P.M. 1994. The moss flora of Mexico. Memoirs of the New York Botanical Garden 69(2): 581-1113.

SUDENE. 1990. Dados pluviométricos mensais do Nordeste: Pernambuco. Série pluviometria 6. Recife.

Vilas Bôas-Bastos, S. B. \& Bastos, C. J. P. 1998. Briófitas de uma área de cerrado no município de Alagoinhas, Bahia, Brasil. Tropical Bryology 15: 101-110.

Vital, D.M.; Giancotti, C. \& Pursell, R.A. 1991. The bryoflora of Fernando de Noronha, Brazil. Tropical Bryology 4: 23-24.

Yano, O. 1981. A checklist of the Brazilian mosses. The Journal of the Hattori Botanical Laboratory 50: $279-456$.

Yano, O. 1984. Briófitas. In: O. Fidalgo \& V.L.R. Bononi (Coords). Técnicas de coleta, preservação e herborização de material botânico. Instituto de Botânica, São Paulo. Manual 4: 27-30.

Yano, O. 1989. An additional checklist of Brazilian bryophytes. The Journal of the Hattori Botanical Laboratory 66: 371- 434 .

Yano, O. 1992. Novas localidades de musgos nos estados do Brasil. Acta Amazonica 22(2): 197-218.

Yano, O. 1995. A new additional annotated checklist of Brazilian bryophytes. The Journal of the Hattori Botanical Laboratory 78: 137-182.

Yano, O. \& Andrade-Lima, D. 1987. Briófitas do Nordeste Brasileiro: Estado de Pernambuco. Revista Brasileira de Botânica 10: 171-181.

Yano, O. \& Bastos, C.J.P. 1994/1995. Musgos do Estado da Bahia, Brasil. Biologica Brasilica 6(1/2): 9-26.

Yano, O. \& Costa, D.P. 1992. Novas ocorrências de Briófitas no Brasil. Pp. 33-45. In: Anais do VIII Congresso da SBSP, Campinas, 1992, Sociedade Botânica de São Paulo.

Yano, O. \& Lisboa, R.C.L. 1988. Briófitas do território federal do Amapá, Brasil. Boletim do Museu Pa- 
raense Emílio Goeldi, Botânica 4(2): 243-270.

Yano, O. \& Mello, Z.R. 1989. Estudos de briófitas do Brasil. 6. Phyllogoniaceae (Bryopsida). Acta Botanica Brasilica 3(2): 119-129.

Yano, O. \& Mello, Z.R. 1992. Briófitas novas para o estado de Roraima, Brasil. Acta Amazonica 22(1): 23-50.

Yano, O. \& Santos, S.X. 1993. Musgos da gruta de Mirassol, São Paulo. Acta Botanica Brasilica 7(2): 89-108. 